\title{
Antitumor Effects and Distribution of Adriamycin Incorporated into Hydroxyapatite Implants in a Cancer Rat Model Bearing Swarm Rat Chondrosarcoma
}

\author{
Keiko Yamamura ${ }^{1}$, Hisashi Iwata ${ }^{2}$, Takashi Osada ${ }^{1}$, Toshihisa Yotsuyanagi ${ }^{3}$ and Toshitaka Nabeshima ${ }^{1}$ \\ ${ }^{\prime}$ Department of Neuropsychopharmacology and Hospital Pharmacy and ${ }^{2}$ Department of Orthopedic Surgery, \\ Nagoya University School of Medicine, Tsuruma-cho, Showa-ku, Nagoya 466, Japan \\ ${ }^{3}$ Faculty of Pharmaceutical Sciences, Nagoya City University, Mizuho-ku, Nagoya 467, Japan \\ Received June 30, 1994 Accepted September 10, 1994
}

\begin{abstract}
We investigated the antitumor effects and tissue distribution of adriamycin (ADR) incorporated into a hydroxyapatite (HAP) bead in a cancer rat model bearing Swarm rat chondrosarcoma. The Porous HAP bead $(8.48 \mathrm{~mm}$ in diameter, $531 \pm 0.7 \mathrm{mg}$ in weight) was used as a model bone graft. One ADR-HAP bead (ADR $0.4 \mathrm{mg}-6.0 \mathrm{mg} / \mathrm{bead}$ ) was implanted s.c. into a Sprague-Dawley rat at 6 days postinoculation of Swarm rat chondrosarcoma. ADR-HAP beads showed strong antitumor activities in a dose dependent manner. The dose of $6.0 \mathrm{mg} / \mathrm{bead}$ showed the highest efficacy with no toxic death: It caused a $98 \%$ growth inhibition on Day 31 postinoculation and a survival advantage of a $339 \%$ increase in life span. After the implantation of the ADR-HAP bead $(0.4 \mathrm{mg} / \mathrm{bead} / \mathrm{body})$ and the i.v. administration of an equal dose of free adriamycin, we determined the tissue distribution of ADR for up to 90 days. ADR-HAP bead implanted in the tumors released ADR over a 12-week period in the target area. The diffusion of the drug to other organs such as the heart and liver was very low compared with the tumors. The area under the ADR concentration-time curve (AUC) of the tumors was $181.6 \mu \mathrm{g} \cdot \mathrm{day} / \mathrm{g}$ and $5.22 \mu \mathrm{g} \cdot \mathrm{day} / \mathrm{g}$ after the implantation of the ADR-HAP bead and the i.v. administration of free ADR, respectively. The targeting index of the tumors, defined as the ratio of the AUC after the implantation of the ADR-HAP bead to that after administration of free ADR, was 34.8. The targeting indices of 0.16 and 0.17 for the heart and liver, respectively, indicate that the implantation of the ADR-HAP bead reduced delivery of ADR to these organs. These results demonstrate that this method of administration may be useful in delivering adjuvant chemotherapy in order to prevent local recurrence in the site of the bony defect after the surgical removal of bone tumors.
\end{abstract}

Keywords: Chondrosarcoma (Swarm rat), Adriamycin, Hydroxyapatite, Tissue distribution

In general, the therapy of malignant bone tumors consists of the combination of en bloc resection with chemotherapy and/or radiation (1). However, bony curettage has its limitations because of the possible persistence of tumor cells. Moreover, there were still unsolved problems with regards to the management of the bony defect and the reduction of the possible toxic side-effects of the antineoplastic agents employed.

Although adriamycin (ADR) is one of the anticancer drugs most widely used against malignant bone tumors (2), its systemic use is often limited due to its cardiotoxic and other undesirable side effects (3). An appropriate drug delivery system that slowly releases an anticancer drug exclusively into the tumor would be promising for both enhancing the therapeutic efficiency for local control of the tumors and for reducing the risk of toxic side effects.

Hydroxyapatite (HAP) is the major mineral component of bone and teeth. Synthetic HAP shows chemical and physical properties similar to those of bone. It has been reported that porous HAP enjoys an excellent biocompatibility with bony tissue, and it can be partly absorbed when used in orthopedics as a replacement substance following local excision with the bony curettage of bone tumors or osteoarthrosis (4). No surgical procedure is required for removal when anticancer drugs are present in a HAP bead. We have reported in a preliminary study that the ADR-loaded HAP bead offers a potentially effec- 
tive method of anticancer drug delivery (5). In the present paper, we describe the dose-dependency of the antitumor effects of an ADR-loaded HAP bead and the tissue distribution of ADR from the HAP bead compared with i.v. administration in rats bearing Swarm rat chondrosarcoma.

\section{MATERIALS AND METHODS}

\section{Chemicals}

ADR injection (Adriacin ${ }^{\mathbb{R}}, 10 \mathrm{mg} /$ vial) and ADR-HCl were supplied from Kyowa Hakko Kogyo Co., Ltd., Tokyo. The porous HAP beads were a gift from NGK Spark Plug Co., Ltd., Nagoya. The physical data of the beads (XVC-56) were as follows: sintering temperature, $1100^{\circ} \mathrm{C}$; diameter, $8.48 \mathrm{~mm}$; weight of one bead, $531 \pm 0.7 \mathrm{mg}$; bulk density, 1.66 ; true density of the material, 2.97; and $\mathrm{Ca} / \mathrm{P}, 1.68$. Distilled water and acetonitrile were of high performance liquid chromatography (HPLC) quality. All other chemicals were of reagent grade.

\section{Animals}

Four-week-old male Sprague-Dawley (SD) rats weighing 85-92 g were obtained from Japan SLC, Ltd., Hamamatsu.

\section{Tumor}

The Swarm rat chondrosarcoma was isolated from a tumor that originally arose spontaneously in a female SD rat (6); histologically, it shared certain features with a well-differentiated (grade I) human chondrosarcoma such as moderate cellularity and mild nuclear hyperchromasia (7). In this study, the Swarm rat chondrosarcoma was maintained by subcutaneous transplantation into SD rats in our laboratory (5). Tumor cell suspensions $\left(10^{7}\right.$ cells $/ \mathrm{ml}$ ) in $0.9 \% \mathrm{NaCl}$ solution were prepared by mincing the surgically obtained tumor through a stainless steel sieve (pore size, $1-\mathrm{mm}^{2}$ ). The tumor pieces were digested for about $90 \mathrm{~min}$ sequentially with $0.5 \%$ trypsin and $0.5 \%$ collagenase. The concentration of cells released was determined by a counter for bio-cells (Elzone ${ }^{\mathbb{B}}$ Model 80; Elmhurst, IL, USA). Tumor cell suspensions (1 ml) were inoculated s.c. into the armpit sites of SD rats on Day 0 . All rats used in a single experiment (6 rats in a group) were inoculated on the same day with cells from the same tumor. When the tumor cell suspension $\left(10^{7}\right.$ cells $)$ was inoculated s.c. into four-week-old SD rats, the tumor size reached about $\sim 1.0 \mathrm{~cm}^{3}$ by 1 week postinoculation and $\sim 196 \mathrm{~cm}^{3}$ by 4 weeks.

\section{Preparation of ADR-HAP beads}

HAP beads were washed with freshly distilled water once a day for a week to remove unknown water-soluble substances and ions and then dried in a desiccator with a vacuum pump and sterilized by autoclaving. The drug amount loaded in the bead was estimated from the mean full displacement of the pore spaces with water $(0.140 \pm 0.001 \mathrm{ml}, \mathrm{n}=10)$. Empty HAP beads were placed in an ADR aqueous solution $(2.86-42.86 \mathrm{mg} / \mathrm{ml})$ at $5^{\circ} \mathrm{C}$ under atmospheric pressure and allowed to remain for 1 hr. HAP beads containing drug solution were lightly wiped with a filter paper and lyophilized in a freezedrying apparatus (8).

\section{Evaluation of antitumor effects}

Free ADR ( $0.4 \mathrm{mg} / \mathrm{body})$ in saline was injected i.v. on Day 6 postinoculation. One ADR-HAP bead $(0.4-6.0$ $\mathrm{mg} / \mathrm{bead} / \mathrm{body}$ ) was implanted into the central portion of the tumor. Drug-free HAP beads were implanted in the same manner to investigate any effects of the HAP beads themselves. Tumor growth rates were determined by measuring the tumor volumes with a caliper every 3 to 4 days postinoculation. The tumor volume $(\mathrm{V})$ was calculated by the formula $1 / 2 a b^{2}$, where $a$ is the long diameter and $b$ is the short diameter. The tumor volume was expressed as the relative tumor volume ( $R V), R V=V / V_{0}$, where $V$ is the tumor volume on the day of measurement, and $V_{0}$ is the initial volume on day 6 (when the treatment was started) (9). The antitumor effect was evaluated by comparing the mean survival time of the treated group (T) with that of the control group (C) and expressing it as "increase in life span" (ILS) or "tumor growth inhibition" on Day 31 postinoculation.

$$
\text { ILS }=(T / C-1) \times 100
$$

\section{Tissue distribution studies}

Rats bearing Swarm rat chondrosarcoma were given either a single i.v. administration of free ADR $(0.4$ $\mathrm{mg} /$ body) or an implantation of ADR-HAP bead ( 0.4 $\mathrm{mg} /$ bead/body) on Day 6. Blood samples $(0.5 \mathrm{ml})$ were taken from the femoral vein at $5,10,20,30,60,180$ and $360 \mathrm{~min}$. The blood was collected in heparinized tubes under ether anesthesia, and centrifuged immediately at $3,000 \mathrm{rpm}$. The plasma supernatants were collected and frozen at $-80^{\circ} \mathrm{C}$ until determination. The excised tumor, heart and liver were rapidly washed with sterilized saline and cut into small pieces in $2 \mathrm{ml}$ of $10 \mathrm{mM}$ phosphatebuffered saline (pH 7.0). These were homogenized with a Polytron homogenizer at $4{ }^{\circ} \mathrm{C}$ to make a $5-10 \%$ homogenate. The homogenate was centrifuged at $12,000 \mathrm{rpm}$ for $5 \mathrm{~min}$. ADR contents in the supernatant was determined by HPLC (10).

\section{Statistical analyses}

Statistical differences between the control and drug-free 
HAP treatment values within the groups were determined by Student's $t$-test, and the differences between the groups were studied by analysis of variance (Scheffe's $F$-test). A $P$ value of $<0.05$ was considered significant.

\section{RESULTS}

\section{Chemotherapeutic effects against Swarm rat chondrosar- coma}

The antitumor effects of local and systemic ADR on this tumor system are shown in Table 1 and Fig. 1. No effect on tumor growth was observed in rats implanted drug-free HAP beads compared with the non-treated control. The ADR-HAP beads showed good therapeutic effects in a dose-dependent fashion. When compared with an equal dose ( $0.4 \mathrm{mg} /$ body) given by systemic administration, the ADR-HAP bead showed more tumor inhibitory effects on Swarm rat chondrosarcoma. The ADR-HAP beads $(0.4 \mathrm{mg} /$ bead) showed chemotherapeutic effects of $69 \%$ ILS and a tumor inhibition rate of $33 \%$, whereas single i.v. injection showed lower effects of $13 \%$ ILS and a tumor inhibition rate of $11 \%$. At the tested doses of ADR
Table 1. Antitumor effects of adriamycin-HAP beads against Swarm rat chondrosarcoma

\begin{tabular}{lcccc}
\hline $\begin{array}{c}\text { Applied form } \\
\text { of drug }\end{array}$ & $\begin{array}{c}\text { Dose } \\
\text { (mg/body) }\end{array}$ & $\begin{array}{l}\text { MST } \\
\text { (days) }\end{array}$ & $\begin{array}{c}\text { ILS } \\
(\%)\end{array}$ & $\begin{array}{c}\text { Tumor growth } \\
\text { inhibition }^{\text {b) }}(\%)\end{array}$ \\
\cline { 1 - 3 } Control & 0 & $31 \pm 2.3^{\text {c) }}$ & & \\
Drug free-HAP & 0 & $32 \pm 2.5$ & & \\
ADR-HAP & 0.4 & $50 \pm 5.2^{*}$ & 69 & 33 \\
ADR-HAP & 1.0 & $58 \pm 8.4^{*}$ & 89 & 69 \\
ADR-HAP & 2.0 & $69 \pm 5.8^{* *}$ & 123 & 82 \\
ADR-HAP & 4.0 & $95 \pm 4.6^{* *}$ & 206 & 90 \\
ADR-HAP & 6.0 & $105 \pm 6.2^{* * *}$ & 339 & 98 \\
ADR-i.v. & 0.4 & $35 \pm 3.1$ & 13 & 11 \\
\hline
\end{tabular}

Swarm rat chondrosarcoma cells $\left(10^{7} /\right.$ rat $)$ were inoculated s.c. into SD rats (6 rats/group) on Day 0, and an ADR-HAP bead was implanted s.c. on Day 6. ADR was administered i.v. on Day 6. ${ }^{\text {a) MST, }}$ mean survival time of deceased time. ${ }^{b}$ Relative tumor growth on Day 31. 'Mean \pm S.E. There was no statistically significant difference between the control group and the drug free-HAP group for MST. ${ }^{\text {d) }}$ Significant differences were observed between ADR-HAP groups ( $\mathrm{P}<0.01$, ANOVA). ${ }^{*} \mathrm{P}<0.05,{ }^{* *} \mathrm{P}<0.01,{ }^{* * *} \mathrm{P}<0.001$, compared to the drug free-HAP (Scheffe's $F$-test).

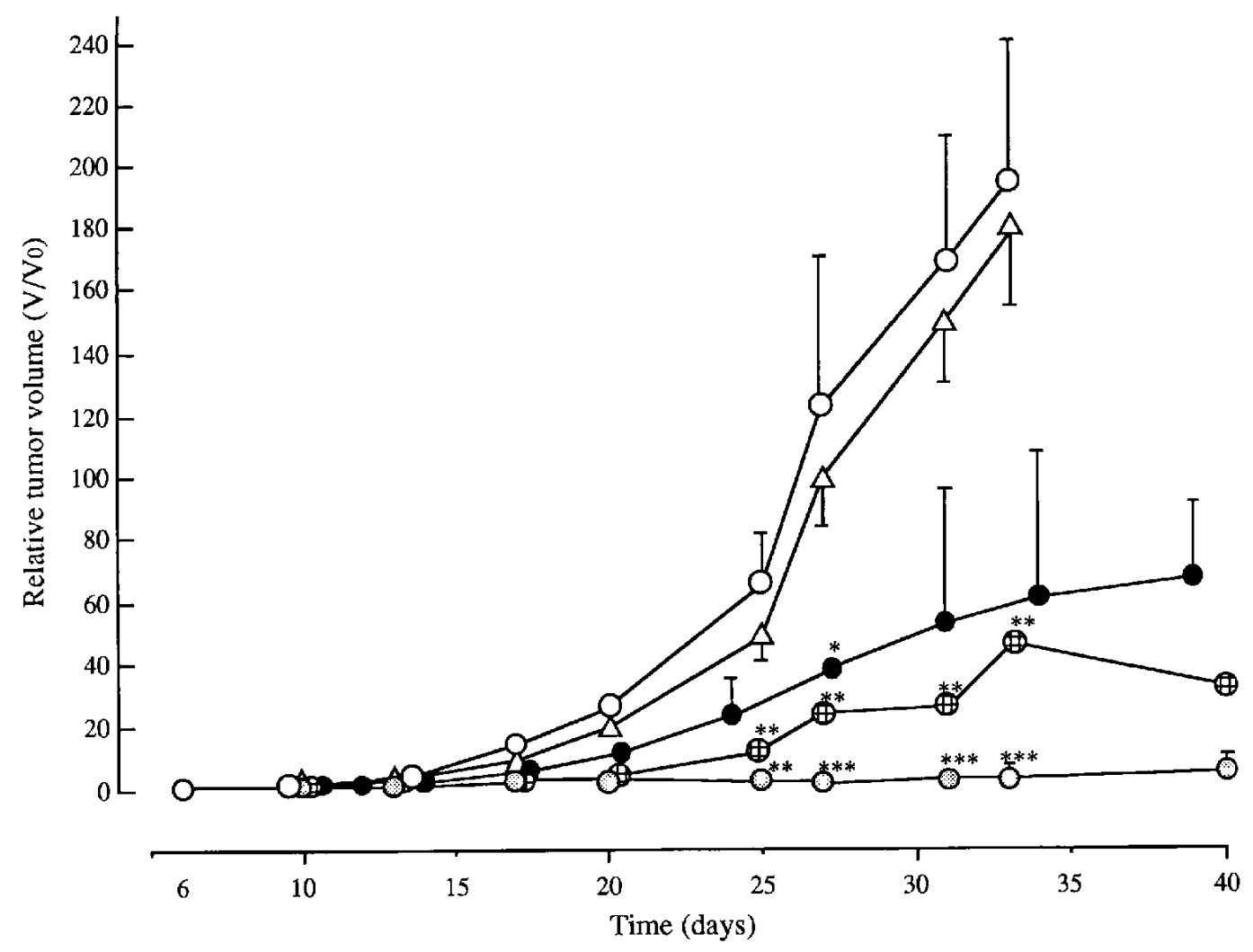

Fig. 1. Antitumor effect of Adriamycin (ADR) on the growth of s.c. Swarm rat chodrosarcoma. $V$ is the tumor volume on a given day, and $\mathrm{V}_{0}$ is the initial volume on day 6 (when the treatment was started). (C) control; (O) ADR-HAP (ADR: 0.4 $\mathrm{mg} /$ body); (๑) ADR-HAP (ADR: $2.0 \mathrm{mg} /$ body); (O) ADR-HAP (ADR: $6.0 \mathrm{mg} /$ body); $\triangle$ ) ADR, i.v. (ADR: $0.4 \mathrm{mg} / \mathrm{body}$ ). Values are expressed as means \pm S.E. $(\mathrm{N}=4-5)$. ${ }^{* * *} \mathrm{P}<0.001,{ }^{* *} \mathrm{P}<0.01,{ }^{*} \mathrm{P}<0.05$ vs control group (Scheffe's $F$-test). 


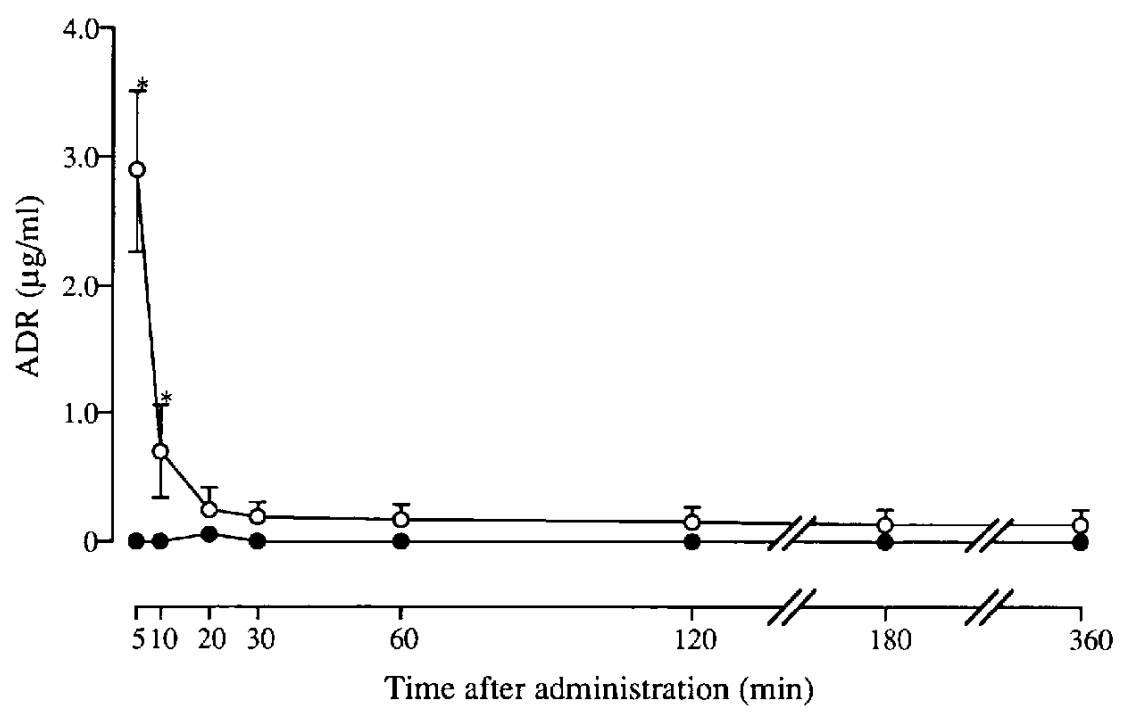

Fig. 2. Adriamycin (ADR) serum levels. One ADR-HAP (ADR: $0.4 \mathrm{mg} / \mathrm{HAP}$ ) bead was implanted in the central portion of the tumor (O). Free ADR $\left(0.4 \mathrm{mg} /\right.$ body) was administered $\mathrm{i} . \mathrm{v}$. (O). Values are expressed as means \pm S.E. $(\mathrm{N}=4-5)$. ${ }^{*} \mathrm{P}<0.05$ vs ADR-HAP bead group (Student's $t$-test).

(4.0 to $6.0 \mathrm{mg} /$ bead), more than $90 \%$ tumor growth inhibition was obtained. The dose of $6.0 \mathrm{mg} /$ bead showed the greatest efficacy compared with the other groups: No toxic death was caused, and there was $98 \%$ growth inhibition on Day 31, and a survival advantage of a $339 \%$ ILS.

\section{$A D R$ levels in serum}

The maximum serum levels of ADR were observed at 5 min after the injection of free ADR, and a rapid clearance of the drug from the circulation was shown (Fig. 2). After the implantation of the ADR-HAP bead, the drug was detected only at very low levels when compared with those associated with i.v. administration.

\section{ADR levels in tumor}

The maximum level of free ADR after i.v. injection of free ADR was $6.7 \mu \mathrm{g} / \mathrm{g}$ at $6 \mathrm{hr}$, and then ADR levels gradually decreased (Fig. 3). Twelve hours after the implantation of the ADR-HAP bead, however, ADR levels in the tumors were observed to be significantly higher than those

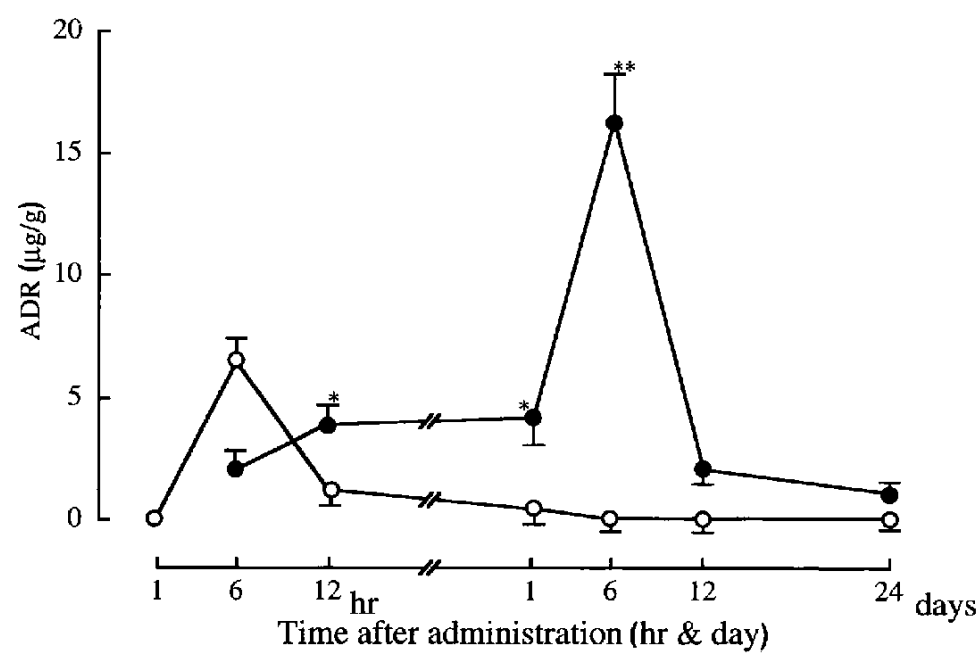

Fig. 3. Adriamycin (ADR) levels in tumors. One ADR-HAP (ADR: $0.4 \mathrm{mg} / \mathrm{HAP}$ ) was implanted in the central portion of the tumor $(O)$. Free ADR $\left(0.4 \mathrm{mg} /\right.$ body) was administered i.v. (O). Values are expressed as means \pm S.E. $(\mathrm{N}=4-5) .{ }^{* *} \mathrm{P}<0.01$, ${ }^{*} \mathrm{P}<0.05$ vs i.v. administration (Student's $t$-test). 
Table 2. AUC values of ADR and targeting index

\begin{tabular}{|c|c|c|c|}
\hline \multirow{3}{*}{ Tissue } & \multicolumn{2}{|c|}{ AUC $(\mu \mathrm{g} \cdot \mathrm{day} / \mathrm{g})^{a)}$} & \multirow{3}{*}{ Targeting index } \\
\hline & implantation $^{\text {b) }}$ & i.v. ${ }^{b)}$ & \\
\hline & ADR-HAP & Free-ADR & \\
\hline Heart & 1.81 & 11.20 & 0.16 \\
\hline Liver & 1.53 & 9.21 & 0.17 \\
\hline Tumor & 181.6 & 5.22 & 34.8 \\
\hline
\end{tabular}

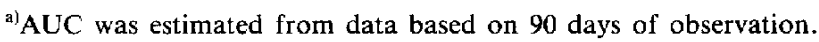
${ }^{b}$ The dose of ADR was $0.4 \mathrm{mg} / \mathrm{body}$. ${ }^{\circ} \mathrm{The}$ targeting index was defined as the ratio of AUC after implantation of the ADR-HAP bead to that after i.y. administration.

following the i.v. administration of free ADR (Fig. 3), and ADR was detectable for up to 90 days. The maximum ADR levels in the tumors after implantation of the ADRHAP bead was $16.2 \mu \mathrm{g} / \mathrm{g}$ at 6 days, 2.4 times higher than the maximum concentration after the i.v. administration of free ADR. The area under the ADR concentrationtime curve (AUC) was $181.6 \mu \mathrm{g} \cdot$ day $/ \mathrm{g}$ after the implantation of the ADR-HAP bead and $5.22 \mu \mathrm{g} \cdot \mathrm{day} / \mathrm{g}$ after the i.v. injection of free ADR. The targeting index was 34.8 for the implantation of the ADR-HAP bead (Table 2). In short, when compared with the i.v. administration, the ADR-HAP bead administration enhanced the delivery of the drug to the tumors.

\section{$A U C$ values of $A D R$ and targeting indices in the heart and liver}

Compared with the AUC values after the i.v. administration of free ADR, those in the heart and liver were low after the implantation of the ADR-HAP bead (Table 2). In the heart, the AUC values was $1.81 \mu \mathrm{g} \cdot \mathrm{day} / \mathrm{g}$ after the implantation of the ADR-HAP bead and $11.2 \mu \mathrm{g}$.day $/ \mathrm{g}$ after the i.v. administration of free ADR. The targeting index was 0.16. In the liver, the AUC values were 1.53 $\mu \mathrm{g} \cdot$ day $/ \mathrm{g}$ after the implantation of the ADR-HAP bead and $9.21 \mu \mathrm{g} \cdot \mathrm{day} / \mathrm{g}$ after the i.v. administration of free ADR. The targeting index was 0.17. AUC values for the 12-24 days after implantation of the ADR-HAP bead were $21.78 \mu \mathrm{g} \cdot \mathrm{day} / \mathrm{g}$ in the tumor, $0.14 \mu \mathrm{g} \cdot \mathrm{day} / \mathrm{g}$ in the liver and $0.15 \mu \mathrm{g} \cdot \mathrm{day} / \mathrm{g}$ in the heart.

\section{DISCUSSION}

ADR is an anticancer drug with broad activity against a number of human neoplasms (2). It is difficult to control local recurrence and pulmonary metastases by i.v. injection after bone tumor surgery. In most cases, hydrophilic anticancer drugs, such as anthracyclinces and mytomycin $C$ used clinically, appear to be rapidly absorbed from the interstitial space through the capillaries, resulting in a low drug concentration at the operated site (11). This was confirmed in the present investigation, because a rapid clearance from the plasma was observed following an i.v. administration of ADR (Fig. 2). It is believed that in comparison with intravenous administration, intra-arterial infusion of anticancer drugs achieves high local leveis of drugs in tumors and reduces systemic toxicity $(12,13)$. This local delivery method may be effective in decreasing the size of the bone tumors, but it cannot be used postoperatively. Therefore, it may be useful to fill up the bony defect after excision with a bone graft incorporated with an active anticancer drug; such an implant would be expected to release active agents for a specific duration. Guan et al. (14) experimented with the incorporation of antineoplastics into bone cement powder for implantation in rat tumors and reported that the antineoplastic effect was higher than for i.v. administration. Blocks, plates or beads are preferable to powder for maintaining bone structure and skeletal support to the body in cases where widespread bone defects exist after surgery for bone tumor. The beads used in our experiment were identical in size and composition and composed of a bone graft used clinically. The number of beads used in each case was sufficient to fill the volume of each bone defect. Therefore, to avoid side effects and achieve chemotherapy, it is important to know the antitumor effects and distribution of the drug in order to predict the amounts of antineoplastics incorporated as the number of beads increases. In our preliminary study, we demonstrated that an ADR-loaded HAP bead is effective when implanted in the Swarm rat chodrosarcoma (5). To explore the usefulness of this method targeting regional chemotherapy on the bone tumors, we investigated in this study the anticancer effects and ADR delivery to various organs in a rat model after the implantation of the ADRHAP bead, in comparison with i.v. administration. Evidence from the present experiments indicate that the drug effects for chemotherapy were well-correlated with the dose of drug incorporated in the HAP bead (Table 1). The dose of $6.0 \mathrm{mg} /$ bead showed the highest efficacy: It showed a $98 \%$ growth inhibition on Day 31 and a survival advantage of a $339 \%$ increase in life span without toxic death. ADR delivery was expressed as the area under the tissue ADR concentration-time curve (Table 2). The AUC in the tumor after the implantation of the ADR-HAP bead was significantly higher than that after the i.v. administration of free ADR. In addition, after the implantation of the ADR-HAP bead, the AUC in the tumors was significantly higher than that in other tissues. The targeting index for the tumors was 34.8 after the implantation of the ADR-HAP bead. The targeting indices for the heart and the liver after the implantation of the ADR- 
HAP bead were very low, indicating that unfavorable ADR delivery to these organs was reduced. ADR was hardly detected in the plasma, except for a very small amount $(0.05 \mu \mathrm{g} / \mathrm{ml})$ at $20 \mathrm{~min}$ after implantation of the ADR-HAP bead (Fig. 2). This ADR delivery system produced a high concentration and a prolonged retention of the drug at the site of the tumor, but a low concentration in the systemic circulation and other organs. These findings indicated that the clinical trials of this system will allow us to control more effectively bone tumors after surgery.

\section{REFERENCES}

1 Dahlin DC and Coventry MB: Osteogenic sarcoma-a study of 6000 cases. J Bone Joint Surg 49, 101-110 (1967)

2 Young $\mathrm{RC}$, Ozols RF and Myers CE: The anthracycline antineoplastic drug. N Engl J Med 305, 139-153 (1981)

3 Minow RA, Benjamin RS and Gottlieb JA: Adriamycin (NSC123127) cardiomyooathy: an overview with determination of risk factors. Cancer Chemother Rep 6, 195-201 (1975)

4 Ono K, Yamamuro T, Nakamura T and Kokubo T: Quantitative study on osteoconduction of apatite-wollastonite containing glass ceramic granules, hydroxyapatite granules and alumina granules. Biomaterials 11, 265-271 (1990)

5 Yamamura K, Iwata H, Osada T, Nabeshima T and Yotsuyanagi $T$ : Anticancer effects of adriamycin-loaded hydroxyapatite implants using a Swarm rat chondrosarcoma model. Jpn
J Pharmacol 65, 289-291 (1994)

6 Maibenco HC, Krehbiel RH and Nelson D: Transplantable osteogenic tumor in the rat. Cancer Res 27, 362-366 (1967)

7 Sanerkin N: The diagnosis and grading of chondrosarcoma of bone. Cancer 45, 582-594 (1980)

8 Yamamura $\mathrm{K}$, Iwata $\mathrm{H}$ and Yotsuyanagi $\mathrm{T}$ : Synthesis of antibiotic-loaded hydroxyapatite beads and in vitro drug release testing. J Biomed Mater Res 26, 1053 - 1064 (1992)

9 Tsuruo T, Sato S and Yusa K: Antitumor activity of ME2303, a fluorine-containing anthracycline, against human tumors implanted in nude mice. Jpn J Cancer Res 80, 686-689 (1989)

10 Masuike T, Odake J, Kohagura M, Noda T and Takemoto $\mathrm{Y}$ : Determination of adriamycin and its metabolites in biological samples using high performance liquid chromatography. II. Analysis of tissue by extraction method. Yakugaku Zasshi 104, 620-623 (1984) (Abstr in English)

11 Takakura $Y$, Matsumoto S, Hashida $M$ and Sezaki $H$ : Enhanced lymphatic delivery of mytomycin $C$ conjugated with dextran. Cancer Res 44, 2505-2510 (1984)

12 Rosen G, Marcove RC, Caparros B, Nirenberg A, Kosloff C and Huros AG: Primary osteogenic sarcoma-the rationale for pre-operative chemotherapy and delayed surgery. Cancer 43 , 2163-2167 (1979)

13 Lee YM: The rationale for intra-arterial chemotherapy. $\mathrm{Br} \mathrm{J}$ Cancer Clin Oncol 23, 1265-1268 (1987)

14 Guan WY, Yi WT and Zhen SS: Experimental research on the use of an antineoplastic drug with a bone implant. Int Orthop 14, 387-391 (1990) 